\title{
The importance of stewardship
}

\author{
Taylor $\mathbf{G}^{1 *}$ \\ ${ }^{1}$ Chief Public Health Officer, Public Health Agency of Canada, Ottawa, ON \\ *Correspondence: Gregory.Taylor@phac-aspc.gc.ca
}

\begin{abstract}
Antimicrobial resistance is a growing public health threat that at times may appear to be unsolvable. However, in the last few years, great advances have been made to address this issue in Canada and around the world. Our Federal Action Plan on Antimicrobial Resistance and Use in Canada has identified three pillars to address antimicrobial resistance: surveillance, stewardship and innovation. In this supplement are examples of successful stewardship programs for the community, physicians and health care organizations; there are many more. Given that we know successful stewardship programs are possible, it is important to continue this work across the country. Each success helps and multiple successes can create a synergistic effect that can lead to a change in patient expectations and prescribing patterns. Our antimicrobials are a precious resource. It is well worth the effort to contribute to this synergy and build a culture of stewardship so that antimicrobials will continue to be effective for generations to come.
\end{abstract}

The World Health Organization has identified that antimicrobial resistance is among the top public health threats of the 21st century (1). The causes of resistance are multiple and complex, and at times solutions may seem elusive. The good news is that we are making progress and advances have been made in decreasing antibiotic use in the last few years - in Canada and around the world. In Canada, a lot of work is underway at local, provincial/territorial and federal levels. The recent Federal Action Plan identifies three pillars to address antimicrobial resistance: surveillance, stewardship and innovation (2). A previous issue of the journal addressed surveillance (3). This issue addresses the importance of stewardship - or the responsible planning and management of antibiotic use.

A major focus of stewardship programs is to identify ways, and support the change needed, to decrease antibiotic use. "Do Bugs Need Drugs", for example, is a community-based education program that includes resources with consistent messages for health care professionals, children and their parents or caregivers and teachers, employers and workers, long term care facilities and the general public. Through common messaging, networking, aligning interests, and finding cost-savings through partnerships and economies of scale, this program has started to document consistent reductions in antibiotic prescribing (4).

Choosing Wisely Canada is a physician-led campaign to engage physicians and patients in conversations about unnecessary care, including antibiotic overuse. Physicians have developed recommendations on actions that can be taken to decrease antibiotic prescribing, as well as evidence-based educational materials to facilitate the important discussion between physicians and patients about the benefits of moderating antibiotic use (5).

Antimicrobial stewardship programs in hospitals have been growing since it became a national requirement for hospital accreditation (6). However, there have been challenges in finding the time and resources to develop and maintain these programs. This issue includes a description of a particularly successful program in Ontario that started with a well-resourced program in an academic hospital which was then leveraged and shared with other academic and community-based hospitals, particularly in intensive care units(7). Regional antimicrobial stewardship communities of practice are now becoming established and there are already initial indicators of success.

The good news is that there appears to be a high level of acceptance of these programs. In a recent Canadian study of critical care physicians, for example, $86 \%$ of respondents agreed that the patients in their ICU benefited 
from an antimicrobial stewardship program and $81 \%$ reported that the program increased their knowledge of appropriate antimicrobial use in the ICU setting (8).

This is simply a sampling of some of the innovative work taking place across Canada to promote antibiotic stewardship. There are many other initiatives that have been equally successful or are underway. This is not to underestimate the challenges that remain in finding the time and resources required to develop successful programs. But it is important to note that there is both an additive and synergistic effect of the work that is underway. Each success helps, and multiple successes over time lead to a change in expectations and prescribing patterns. It may be gradual at first, but then a momentum is created, and a "new normal" is established.

Antimicrobial resistance will be with us for some time to come, but we are making strides. Each of us has a role to play in helping to advance stewardship. By combining our efforts we can help to ensure that antimicrobials continue to be effective for many generations to come.

\section{Conflict of interest}

None

\section{References}

(1) World Health Organization (WHO). Antimicrobial Resistance-Global Report on Surveillance, 2014. Geneva: WHO; 2014.

http://apps.who.int/iris/bitstream/10665/112642/1/9789241564748_eng.pdf?ua=1

(2) Public Health Agency of Canada. Summary of the Federal Action Plan on Antimicrobial Resistance and Use in Canada: Building on the Federal Framework for Action. CCDR Suppl. 2015 Jun;41(S-4):18-21. http://www.phac-aspc.gc.ca/publicat/ccdr-rmtc/15vol41/dr-rm41s-4/index-eng.php

(3) Can Comm Dis Rep. Antimicrobial resistance Volume 40 S-2, 2014. http://www.phac-aspc.gc.ca/publicat/ccdrrmtc/14vol40/dr-rm40s-2/index-eng.php

(4) Carson M, Patrick DM. "Do Bugs Need Drugs?" A community education program for the wise use of antibiotics. CCDR Suppl. 2015 Jun;41(S-4):4-7. http://www.phac-aspc.gc.ca/publicat/ccdr-rmtc/15vol41/dr-rm41s-4/index-eng.php

(5) Born KB, Leis JA, Gold WL, Levinson W. "Choosing Wisely Canada" and antimicrobial stewardship: A shared focus on reducing unnecessary care. CCDR Suppl. 2015 Jun;41(S-4):8-12. http://www.phac-aspc.gc.ca/publicat/ccdr-rmtc/15vol41/dr-rm41s-4/index-eng.php

(6) Accreditation Canada. Required Organizational Practices Handbook 2014 Antimicrobial stewardship p.33. https://www.accreditation.ca/sites/default/files/rop-handbook-2014-en.pdf

(7) Nakamachi Y, West S, Dresser L, Morris AM. Developing and expanding hospital antimicrobial stewardship: The Ontario experience. CCDR Suppl. 2015 Jun;41(S-4):13-17. http://www.phac-aspc.gc.ca/publicat/ccdr-rmtc/15vol41/dr-rm41s-3/index-eng.php

(8) Steinberg M, Dresser LD, Daneman N, Smith OM, Matte A, Marinoff N, Bell CM, Morris AM. A national survey of critical care physicians' knowledge, attitudes, and perceptions of antimicrobial stewardship programs. J Intensive Care Medicine. 2014 Jul 8. doi:10.1177/0885066614541922 